\section{Óbitos neonatais: por que e como informar?}

\author{
Neonatal death; why and how to inform?
}

Linda Délia Carvalho de Oliveira Pedrosa 1 Silvia W. Sarinho 2 Manoelina de Albuquerque Rocha Ordonha 3

\begin{abstract}
The neonatal death constitutes the major component of the infant mortality (MI) in Brazil, having the asphyxia, the low birthweight, the newborn respiratory affection, the infection and prematurity as the main causes. These situations connect to the prevention failures so the wide determinant network of these obits need to be recognized from available data by national systems of information. A good system covering, agility and reliable information collection in all the levels and the flow information control, assure the validity of the produced indicators, allowing the choice of the best preventive approach. This article presents the several research sources in neonatal obits, their uses and limitations, stooding out the health area professionals contribution of reliable information production. A review on this issue in health ministry technical manuals, theses, dissertations and books, as well as in the electronic data bases Medline, LILACS, SCIELO and PAHO was carried out. Aricles published from 1980 thru 2004, with the following key-words, were included: "infantile mortality", "neonatal mortality", "precocious neonatal mortality", "basic cause of death", "information systems", "information in health", "medical registers", "mortality registers".
\end{abstract}

Key words Infant mortality, Death certificates, Information systems, Medical records

\section{Resumo}

Os óbitos neonatais se constituem no mais importante componente da mortalidade infantil (MI) no Brasil, tendo como principais causas a asfixia, o baixo peso ao nascer, as afecções respiratórias do recém-nascido, as infecções e a prematuridade. São situações vinculadas a falhas de prevenção e a ampla rede de determinantes desses óbitos precisa ser conhecida a partir das informações disponibilizadas pelos sistemas nacionais de informação. Uma boa cobertura do sistema, agilidade e coleta de informações confiáveis em todos os níveis e o controle do fluxo dessas informações, asseguram a validade dos indicadores gerados, permitindo a escolha da melhor abordagem preventiva. Neste artigo, são apresentadas as diversas fontes de pesquisa em óbitos neonatais, seus usos e limitações ressaltando o papel dos profissionais de saúde na geração de dados confiáveis. Foram consultados manuais técnicos do Ministério da Saúde, teses, dissertações e livros sobre o tema e realizada uma revisão nos bancos de dados eletrônicos Medline, LILACS, SCIELO e PAHO em artigos disponíveis de 1980 a 2004, a partir dos descritores "mortalidade infantil", "mortalidade neonatal", "mortalidade neonatal precoce", "causa básica de óbito", "sistemas de informação", "informação em saúde", "registros médicos", "registros de mortalidade".

Palavras-chave Mortalidade Neonatal. Atestados de óbito. Sistemas de informação. Registros médicos 


\section{Introdução}

O nascimento de uma criança desperta alegrias, esperanças e responsabilidades sociais. Contrariando a evolução natural, o óbito infantil fere a sociedade, desnudando, como um sensível indicador, o grau de desenvolvimento e as condições de vida que prevalecem em uma dada população. ${ }^{1}$ A saúde da criança, organismo em fase de crescimento e desenvolvimento, é muito sensível aos agravos de condições externas de caráter socioeconômicas, do ambiente físico e emocional, e da assistência à saúde. ${ }^{1}$

As taxas de mortalidade infantil (MI) variam entre as regiões do mundo e refletem as desigualdades sociais e econômicas existentes. Seu declínio tem aprofundado estas diferenças. ${ }^{2}$ Seguindo essa tendência, o Brasil vem reduzindo significativamente a MI que se concentra, desde o final do século $\mathrm{XX}$, no período perinatal, onde as causas de óbito são de mais difícil redução, por serem menos sensíveis a ações globais na melhoria da qualidade de vida. 3,4

Este artigo se propõe a rever a literatura acerca da mortalidade neonatal no Brasil, enfatizando sua importância no contexto atual da MI, seus determinantes, e a importância da utilização da informação de mortalidade para o sistema de saúde. Foram consultados manuais técnicos do Ministério da Saúde, teses, dissertações e livros sobre o tema e realizada uma revisão nos bancos de dados eletrônicos Medline, LILACS, SCIELO e PAHO em artigos disponibilizados a partir de 1980, partindo-se dos descritores "mortalidade infantil", "mortalidade neonatal", "mortalidade neonatal precoce", "causa básica de óbito", "sistemas de informação", "informação em saúde", "registros médicos", "registros de mortalidade".

\section{Por que é importante informar?}

As políticas de assistência materno-infantil no Brasil foram decisivas para a expressiva redução ${ }^{5}$ do componente pós-neonatal da MI, ao instituírem ações de controle das doenças diarréicas e das infecções respiratórias, além de promoverem ações de expansão da assistência pré-natal, incentivo ao aleitamento materno e priorizarem as campanhas de vacinação. A expansão do saneamento básico na década de 1980 e, mais recentemente, a queda da fecundidade também contribuiram de forma substancial na queda da MI no país. Contudo, a MI ainda é um grave problema de saúde pública no Brasil,6 ainda distante dos níveis preconizados como mode- rados pela Organização Mundial de Saúde (OMS), de cerca de 18 óbitos por mil nascidos vivos. Essa realidade, muito aquém das nossas possibilidades, reflete a relevância dos nossos problemas sociais, perpetuando as acentuadas diferenças regionais. 4

Os Indicadores e Dados Básicos para a Saúde de $2003^{4}$ apontam para taxas de MI estimadas pelo Instituto Brasileiro de Geografia e Estatística (IBGE) em 2001, de 27,4 por 1000 nascidos vivos para a região Norte, 18,2 por mil nascidos vivos para a região Sudeste, 20,9 por 1000 nascidos vivos para a região Centro-Oeste e 43 por 1000 nascidos vivos para o Nordeste. A magnitude da MI e de seu principal componente, a mortalidade neonatal, é maior nas regiões Norte e Nordeste, onde também é menor o acesso aos cuidados de saúde de qualidade no período perinatal. 1

No Brasil, as principais causas de óbitos neonatais são a asfixia intra-uterina e a intraparto, o baixo peso ao nascer, as afecções respiratórias do recém-nascido, as infecções e a prematuridade. São situações vinculadas a falhas de prevenção, exigindo melhoria do acesso, qualidade e da utilização dos serviços de saúde e na distribuição equânime dos recursos. 5 A disponibilização à população geral dos avanços tecnológicos e a regionalização da atenção à saúde obstétrica e neonatal foram essenciais na redução da mortalidade neonatal ocorridos nos países desenvolvidos onde predominam como causas de óbitos neonatais a prematuridade extrema e as malformações congênitas, traduzindo-se em diminuição dos patamares de mortalidade neonatal a partir dos quais a redução é lenta.7

A MI resulta de uma complexa rede de determinantes e, apesar do reconhecimento do papel dos determinantes distais como a pobreza, refletidos no ambiente hostil e na baixa escolaridade materna, o enfoque nos fatores proximais permite o direcionamento das ações do setor saúde, aqueles passíveis de intervenção. ${ }^{7}$ A prioridade no conhecimento da estrutura de determinação do óbito neonatal é imprescindível para todos os níveis de atenção ao neonato, possibilitando que as informações não fiquem estanques. Desse modo, as informações acerca de ações desencadeadas no pré-natal devem chegar ao conhecimento dos assistentes ao parto, otimizando a utilização de recursos e subsidiando a assistência, além de, ao final, alimentar os sistemas de informações estatísticas, redes de pesquisas e orientar a proposição de protocolos clínicos.

Em todas as etapas de cuidados ao neonato que iniciam no pré-natal e culminam com a assistência nas unidades de terapia intensiva podem ser encontrados fatores que podem ser apontados e conside- 
rados como determinantes da mortalidade neonatal. Araújo et al. ${ }^{8}$ detectaram que $30 \%$ dos óbitos poderiam ser evitados através ações desencadeadas no pré-natal, acompanhamento durante o parto e assistência adequada ao recém-nascido na sala de parto. Dentre essas situações passíveis de redução e controle, estão incluídas as infecções, hipertensão e diabete materna, a asfixia perinatal e as infecções do recém-nascido. 4,5

A atenção pré-natal adequada pode reduzir a mortalidade neonatal ao detectar e tratar doenças maternas, promover um manejo nutricional adequado à gestante, propiciar vacinação contra o tétano, além de permitir o aconselhamento materno e a detecção precoce de fatores que poderão vir a contribuir para o adoecimento e morte dos bebês. 8 Pesquisas brasileiras reforçam a necessidade da qualidade da assistência pré-natal, precocidade no seu início, realização de exames complementares, além do acesso fácil, baixo custo e não apenas quantidade adequada de consultas. $9-11,13$

Em relação às características maternas e dos neonatos, a idade materna também é fator importante na mortalidade neonatal. Segundo a Organização Panamericana de Saúde (OPAS), 12 os grupos etários extremos, mulheres menores de 20 e maiores de 35 anos de idade, apresentam mais elevado risco de mortalidade materna e neonatal. A associação entre mortalidade neonatal e peso ao nascer foi demonstrada em diversos estudos, com elevada concentração de óbito entre as crianças de baixo peso, onde o risco chegou a ser $46 \%$ superior às demais faixas ponderais.13 Silva e Ribeiro 9 alertam para um aumento de nascimentos de recém-nascidos de baixo peso em regiões de melhor acesso à assistência médica e onde intervenções médicas mais precoces e adequadas são responsáveis pela sobrevida de crianças mais prematuras, sugerindo que a taxa de recém-nascidos de baixo peso não seja mais sistematicamente usada como indicador de desenvolvimento social.

A menor duração da gestação também contribui com risco de óbito, sendo esse quase 50 vezes maior para as crianças com menos de 28 semanas de gestação, 13 tornando a prematuridade um dos fatores mais importantes para a mortalidade neonatal. A prematuridade e as infecções neonatais, além de contribuirem para a elevação dos índices de mortalidade neonatal, comprometem a qualidade de vida dos sobreviventes, com pesado ônus familiar e social. ${ }^{14-18}$

A gemelaridade esteve relacionada com o baixo peso, prematuridade e mortalidade neonatal no estudo de Bercini,15 confirmando achados de
Guaschino, 16 que afirmou ser o parto prematuro uma complicação típica da gravidez dupla, predispondo ao aumento da mortalidade perinatal e neonatal, principalmente em menores de 34 semanas de idade gestacional.

$\mathrm{O}$ atendimento inadequado ao parto é outro fator determinante para o aumento da mortalidade neonatal. No Brasil $90 \%$ dos nascimentos são hospitalares, mas Araújo et al. ${ }^{8}$ chamam a atenção para o grande número de crianças nascidas em hospitais ligados ao Sistema Único de Saúde (SUS) sem pediatra em sala de parto, o que é uma exigência do Ministério da Saúde, desde 1993. A Academia Americana de Pediatria estima que 5 a $10 \%$ de todos os bebês que nascem, necessitam de alguma manobra de reanimação e a adequada e pronta reanimação desses bebês colabora para reduzir o percentual de $20 \%$ dos óbitos em todo o mundo por asfixia perinatal. 17

O estudo de Coutinho18 é um dos muitos que demonstram a maior ocorrência dos óbitos neonatais no primeiro dia de vida, especialmente nas primeiras horas de vida, reforçando a sua relação com a assistência recebida no período perinatal. A atenção adequada apenas ao parto não é suficiente para reduzir a mortalidade neonatal. São inúmeros os avanços tecnológicos inseridos no cuidado neonatal nas últimas décadas e que foram responsáveis por um aumento considerável do número de neonatos sobreviventes; dentre eles se destacam o maior conhecimento sobre a fisiologia do prematuro, a mecânica ventilatória no período neonatal, o surgimento da terapia de reposição de surfactante exógeno e a adequação dos conhecimentos em nutrição parenteral.19,20

De alto custo, essa tecnologia disponível nas Unidades de Terapia Intensiva Neonatal (UTIs) ainda não está acessível a toda a população brasileira. Nas maternidades das regiões menos favorecidas os berçários são mal equipados, há pouco pessoal treinado, a área física em geral é restrita. Faltam leitos e sobram pacientes que superlotam as unidades, aumentando os riscos de infecções e o obituário, que muitas vezes se desloca do período neonatal precoce para o período neonatal tardio, não sem ônus financeiro, social e emocional. Por outro lado, Araújo et al. 8 também salientam que a sofisticação tecnológica das UTI neonatais prolonga artificialmente a vida de recém-nascidos muito graves e com poucas chances de sobrevivência, contribuindo para a redução da mortalidade precoce, sem que isso signifique melhoria das condições de atendimento à gestante e ao recémnascido. 
As situações de atenção à saúde de cada região do Brasil guardam características particulares que precisam ser bem conhecidas no âmbito local. Devem transformar-se de simples dados em informações confiáveis e válidas, que possam ser coletadas, processadas e analisadas de forma oportuna. Sua fidelidade deve permitir subsidiar as ações dos gestores nas diversas esferas de poder de decisão.

\section{Como e onde obter informações acerca de óbitos neonatais?}

A informação é uma ferramenta essencial à tomada de decisões em todos os âmbitos da vida humana. $\mathrm{Na}$ área da saúde, a simples existência de um dado isolado apenas nos permite especulações que carecem de valor científico e de confiabilidade, o que pode lhe destituir de toda a relevância. Os registros rotineiramente produzidos nas diversas atividades de saúde são os dados que podem ser transformados em informações. Ao se consolidarem em um conjunto integrado de componentes articulados, dentro de regras determinadas e um referencial explicativo sistemático, levam a produção e análise de indicadores, incorporados nos Sistemas de Informação em Saúde (SIS).

Os SIS são parte do SUS que se propõem a organizar a produção de informações, respaldar as atividades diárias, diagnóstico e tratamento, conhecer e monitorar o estado de saúde da população. Com essas informações o SIS pretende facilitar o planejamento, supervisão e controle de ações e serviços, subsidiar os processos decisórios, apoiar a produção e utilização de serviços de saúde. São ainda seus objetivos avaliar intervenções, resultados e impacto, subsidiar educação, promoção de saúde, pesquisa e produção de conhecimento. ${ }^{21}$

Os SIS devem ser capazes de medir a eficácia, efetividade e eficiência do SUS através da geração de indicadores simples, válidos, disponíveis, robustos, sintéticos. Com boa cobertura e poder discriminatório podem permitir ao SUS alcançar os objetivos de integralidade de assistência, descentralização político administrativa, divulgação de informações em saúde e controle social22. É importante a equipe de saúde estar consciente do seu papel na produção das informações que subsidiam e mantém o funcionamento adequado dos SIS.

É evidente o papel da informação em saúde e da dimensão que a estatística tem na construção e disponibilização de indicadores que permitam racionalizar a aplicação de recursos e direcionar ações. 22,23 Uma boa cobertura do sistema, agilidade e coleta de informações confiáveis em todos os níveis e o controle do fluxo das informações, asseguram a validade dos indicadores gerados.

Existem várias formas de se obter informações sobre mortalidade no Brasil, dentre as oficiais, algumas gerenciadas pelo IBGE e outras pelo Ministério da Saúde. O IBGE é o órgão responsável pelas informações estatísticas nacionais, executando censos, inquéritos e pesquisas por amostras domiciliares a partir das quais são geradas as bases populacionais usadas no cálculo de indicadores. As estimativas de MI incluindo a mortalidade perinatal do IBGE são baseadas em métodos demográficos indiretos, prática que gera distorções duramente criticadas pelos gestores, na medida em que são incapazes de detectar mudanças de curto prazo, decorrentes de intervenções na área de saúde.1,6

O Sistema de Informação sobre Nascidos Vivos (SINASC) e o Sistema de Informações sobre Mortalidade (SIM), ambos gerenciados pelo Ministério da Saúde, fazem parte dos SIS de abrangência nacional, possibilitando análises comparativas entre os diversos estados, municípios e regiões. O SIM, instituído em 1975, é o mais antigo dos SIS do Brasil e todas as informações relativas aos óbitos, são obtidas através do preenchimento da Declaração de Óbito (DO), documento padronizado em todo o território nacional, composto de nove blocos de informações com sessenta e duas variáveis. 24

A DO deve ser preenchida pelo médico por ocasião do evento ou pelo Serviço de Verificação de Óbitos (SVO) em óbitos naturais sem assistência médica, ou ainda pelos Cartórios do Registro Civil em localidades sem médico.25 É impressa em três vias pré-numeradas, cujo fluxo varia de estado para estado, sendo recomendação do Ministério da Saúde que a primeira seja recolhida pelas secretarias municipais e codificadas as causas de óbito, suas informações transferidas dos municípios aos estados. Alimentam o banco de dados nacional do SIM, que as disponibiliza em parte pela Internet no site do DATASUS; a segunda via entregue pela família ao cartório de registro civil serve para a emissão da Certidão de Óbito e procedimentos legais de sepultamento e a terceira via deve permanecer nos hospitais. 24,25

A cobertura do SIM atingiu $83 \%$ em 2002, mas é bastante heterogênea, variando de $68,4 \%$ no Nordeste e $94,4 \%$ no Sul do país. Também existe heterogeneidade na qualidade de suas informações, mantendo um percentual de causas mal definidas de $12,4 \%$ (26,7\% no Nordeste e 6,3\% no Sul). 26 A subnotificação de eventos, percentual excessivo de causas mal definidas de óbito, ou erros de classifi- 
cação de óbitos fetais e não fetais, além do preenchimento incorreto ou incompleto da DO, resultam em variações na qualidade do SIM. ${ }^{4}$ Dados inconsistentes, sub-estimativa das causas maternas e informações tidas como "ignoradas" também aumentam a ineficiência do sistema de informação. 27,28

Alguns autores18,29 têm questionado se os motivos que levam a deficiência no preenchimento das declarações de óbitos por parte dos médicos não decorreriam da pouca ênfase dada aos alunos na graduação sobre a importância de seu preenchimento correto ou da pouca qualificação, desconhecimento da utilidade da DO, vendo-a apenas como documento legal,18,29,30 e até mesmo desinteresse pelo assunto.

Além dos problemas relacionados ao preenchimento incorreto ou incompleto e a existência de informações não conclusivas nas declarações de óbito, outros entraves dificultam a sua qualificação como fonte de informações de relevância: as digitações incompletas nos órgãos competentes, o grande número de variáveis "ignoradas", pequeno controle sobre documentos incompletos ou inconsistentes vindos dos serviços hospitalares e a falta de legibilidade da caligrafia dos médicos, comprometendo o correto diagnóstico e codificação da causa básica. $27-29$

O SINASC, concebido à semelhança do SIM e implantado a partir de 1990, é outro SIS que recolhe informações a partir da Declaração de Nascido Vivo (DNV), padronizada e de distribuição gratuita pelo Ministério da Saúde em todo o país. Dados do DATASUS de 2001 estimavam uma cobertura para o SINASC em todo o Brasil da ordem de 93\%, superando amplamente a de nascimentos registrados em cartórios. Em 2002 esse percentual foi reduzido no Brasil para $85,4 \%$, mantendo-se heterogêneo com $94,5 \%$ no Centro-Oeste e $80,3 \%$ no Norte e Nordeste. 26,30 . Seria importante a análise em conjunto dos Sistemas SIM e SINASC permitir a geração de indicadores para o monitoramento da MI e da moralidade neo e perinatal, mas as dificuldades de cobertura e a baixa qualidade dos dados disponibilizados ainda impossibilitam a produção e comparabilidade desses indicadores 9,26 . No sentido de melhorar as inforamções nesta área a técnica conhecida como linkage quando utiliza bancos de dados secundários 31 e é um procedimento metodológico que tem sido bastante empregado em pesquisas brasileiras. 5,8,9,13,32,33 É utilizado rotineiramente em países como os Estados Unidos, ${ }^{34}$ que também encontram dificuldades relativas à qualidade da informação médica. A técnica de linkage de bancos de dados do SIM/SINASC permite estudar fatores de risco para óbitos neonatais e avaliar a influência do peso, fatores sócio-demográficos e assistenciais na mortalidade neonatal.5,8,9,11,13,18

No intuito de restringir as distorções decorrentes do sub-registro de nascimentos e de óbitos, nos indicadores gerados a partir dessas informações de qualidade limitada, pesquisas realizadas contemplando as diversas regiões brasileiras, utilizaram outras fontes de dados além da DNV e DO: dados censitários, o Sistema de Informação Hospitalar (SIH) do SUS, informações colhidas nos livros de anotações das maternidades, prontuários médicos e até em cemitérios, além de inquéritos, autópsia verbal e pesquisas qualitativas com uso de entrevistas e visitas domiciliares. 5,8,9,13,32,35-38

O prontuário médico, conjunto de documentos padronizados e ordenados, destinados ao registro dos cuidados profissionais prestados ao paciente, é um elemento valioso para o paciente e a instituição que o atende, para o médico, bem como para o ensino, a pesquisa e os serviços de saúde. ${ }^{39}$ Estudos em diferentes estados e regiões brasileiras apontam para uma deficiência permanente na qualidade dos prontuários médicos. Diversos estudos brasileiros utilizaram registros e prontuários médicos na análise da causa básica de óbito neonatal.18,29,38 Hartz et al.,40 em 1996, enfatizam a observação de Potvin, em 1984, de que a validade da informação dos óbitos aumenta com a revisão de prontuários. Formigli $e t$ al., 41 em Salvador, estado da Bahia, obtiveram um percentual de perdas de informações de 58,3\% decorrentes da não localização de prontuários nas unidades hospitalares e falta de legibilidade, além da inexistência de registro de exame físico e até do tratamento.

Santa Helena e Rosa42 ressaltaram a "pobreza" de informações, relativas aos óbitos de crianças menores de um ano em Blumenau, dificultando a avaliação do cuidado e a compreensão dos eventos que levaram a morte. Para melhoria na qualidade dos prontuários médicos os autores recomendam reuniões periódicas de revisão de casos, orientação também preconizada por Coutinho. ${ }^{18}$

Conscientes de que nenhum tipo de coleta de dados é totalmente isenta de vícios ou dificuldades de realização e, para minimizar a insuficiência das informações, alguns pesquisadores optaram pela complementação das informações utilizando-se de outras fontes disponíveis, como os registros hospitalares, livros de admissão e alta, além de resultados de necrópsias. Barros et al. 43 investigaram, em 1985, os óbitos de uma coorte de crianças nascidas em Pelotas, no Rio Grande do Sul, através de visitas domiciliares e revisão de prontuários médicos. 
Dados de necrópsia são de difícil obtenção, mesmo quando os estudos são preparados para a sua realização, pela dificuldade relativa a necrópsia na faixa etária neonatal e em razão da pouca aceitação dos familiares em sua autorização. 44

Algumas pesquisas no Brasil utilizaram entrevistas com mães e profissionais envolvidos na assistência, como alternativa na obtenção de dados e incluíram, também, a análise qualitativa da assistência, grau de satisfação dos profissionais envolvidos na atenção médica e enfatizaram o grau de satisfação da mãe pela assistência recebida e suas próprias conclusões sobre as causas de óbito dos seus filhos.35,45,46 Schramm e Szwarcwald,47 utilizando o Sistema de Informação Hospitalar (SIH/SUS) através da análise dos formulários de Autorização de Internamento Hospitalar (AIH), concluíram pela contribuição dessa fonte de dados devido à agilidade em disponibilizar informações em meio magnético. No entanto, o SIH/SUS não dispõe de condições de obter informações detalhadas de cada caso, devido à própria natureza e função do documento oficial que é a Autorização para Internamento Hospitalar (AIH).

Em 2001, Mello-Jorge e Gotlieb 48 propuseram a utilização do Sistema de Informação Básica (SIAB) vinculado ao Ministério da Saúde como forma alternativa de complementar as informações do SIM e SINASC, para redução do sub-registro nestes sistemas, dada a maior proximidade dos agentes comunitários com a ocorrência dos eventos, sendo os mesmos uma excelente fonte de informações sobre eventos vitais. Também Victora e Tomasi, 49 em 2004, destacaram a qualidade do SIAB, sendo conveniente atentar para as falhas dessa fonte em locais de baixa cobertura pelo sistema. A utilização dos dados relativos ao registro civil encontra uma séria limitação no subregistro dos óbitos bem como no atraso do registro ou sub enumeração dos nascimentos, condicionados pelo desconhecimento da importância do seu registro pela população.

Uma forma sistematizada de diagnóstico local da situação de mortalidade infantil e seus componentes pode ser obtida pela instituição rotineira da investigação de óbitos, que deveria ser uma iniciativa de cada serviço de assistência à criança. Como parte das ações de enfrentamento aos elevados índices de mortalidade infantil no país, foi lançado, em 2004, o Pacto Nacional pela Redução da Mortalidade Materna e Neonatal, como prioridade do Ministério da Saúde e que tem como uma das ações estratégicas a vigilância dos óbitos infantis, através da investigação sistemática de todos os óbitos ocorridos a partir de sua identificação, qualificação das informações e avaliação da assistência prestada pelos profissionais no seu dia-a-dia, com o propósito de identificar e prevenir a ocorrência de óbitos evitáveis. 50 É também uma estratégia que visa qualificar as informações disponibilizadas concorrendo para a melhoria de preenchimento dos documentos oficiais.

\section{Considerações finais}

Envolvidos num cotidiano de constantes desafios, em níveis diversos de complexidade, os profissionais de saúde desconhecem ou subestimam sua capacidade de gerar informações que permitam conhecer e analisar a realidade local. A construção de indicadores de qualidade, subsidiando o planejamento de ações e de políticas de saúde eficazes, depende do reconhecimento pelo profissional do seu papel na geração das informações, tornando-as fiéis às necessidades específicas. 30

A melhoria dos sistemas de informação está condicionada a esse reconhecimento e no investimento em capacitação dos profissionais, além do retorno dos resultados das análises produzidas, retroalimentando os setores geradores da informação.

Ainda é atual, pertinente e relevante a afirmação de Aerts (1997: 365)27

"a investigação de sub-registro e a busca da qualificação do preenchimento da declaração de óbito são contribuições imprescindiveis para a construção de um sistema de saúde que atenda as reais necessidades de sua população. Sistema esse que, pautado pela busca da equidade, deverá ser capaz de identificar grupos populacionais em maior risco, dirigindo-lhes uma atenção diferenciada".

As fontes de informação são instrumentos fundamentais na identificação e acompanhamento dos problemas de saúde das populações. O papel a ser desempenhado pelos profissionais da área na geração desses dados com validade e confiabilidade e na sua análise criteriosa deve ser refletido para melhor agilidade do sistema de saúde, com resultados mais efetivos. 


\section{Referências}

1. Szwarcwald CL, Leal MC, Castilho EA. Mortalidade infantil no Brasil: Belíndia ou Bulgária? Cad Saúde Pública 1997; 13: 503-16.

2. Desigualdades en la mortalidad infantil en la región de las Américas: elementos básicos para el análisis. Bol Epidemiol Organ Panam Saúde 2001; 22: 4-7.

3. Darmstadt GL, Lawn EJ, Costello A. Advancing the state of the world's newborn. Bull World Health Organ 2003; 81: 224-5.

4. Ministério da Saúde, OPAS (Organização Pan-Americana de Saúde). Brasil: indicadores e dados básicos para a saúde, 2003.

5. Leite AJM. Mortalidade perinatal e aspectos da qualidade da atenção à saúde no município de Fortaleza, 1995 [dissertação Mestrado]. São Paulo: Departamento de Epidemiologia da Escola Paulista de Medicina, Universidade Federal de São Paulo; 1996.

6. Lansky S, França E, Leal MC. Mortalidade perinatal e evitabilidade: revisão da literatura. Rev Saúde Pública 2002; 36: 759-72.

7. Jones G, Steketee RW, Black RE, Zulfiqar AB, Morris SS, Bellagio Child Survival Study Group. How many child deaths can we prevent this year. - child survival II. Lancet 2003; 362: 65-71.

8. Araújo BF, Bozetti MC, Tanaka ACA. Mortalidade neonatal precoce no município de Caxias do Sul: um estudo de coorte. J Pediatr [Rio J] 2000; 76: 200-6.

9. Silva AAM, Ribeiro VS. Avaliação da qualidade dos dados do Sistema de Informações sobre Nascidos Vivos em 1997. 1998. Rev Saúde Pública 2001; 35: 508-14.

10. Menezes AMB, Barros FC, Victora CG, Alves C, Rocha C, Albenaz E, Matijasevich A, Domingues MR, Sclowitz IKT, Hallal PC, Silveira MF, Vauahan JP. Mortalidade perinatal em duas coortes de base populacional no Sul do Brasil: tendências e diferenciais. Cad Saúde Pública 1996, 12 (Supl. 1): 33-41.

11. Trevisan MR, De Lorenzi DRS, Araújo NM. Perfil da assistência pré-natal entre usuárias do Sistema Único de Saúde em Caxias do Sul. Rev Bras Ginecol Obstet 2002; 24: 293-9.

12. OPAS (Organización Panamericana de la Salud). Las condiciones de salud de las Americas. Washington (DC); 1990 (Publicaciones Cientificas, 524).

13. Rodrigues EM. Estimativas de riscos de mortalidade neonatal a partir dos dados das Declarações de Nascimento e Óbito: estado de São Paulo, Brasil [tese doutorado]. São Paulo: Departamento de Nutrição da Escola Paulista de Medicina da Universidade Federal de São Paulo; 2003.

14. Sarinho SW. Fatores de risco para óbitos neonatais no Recife: um estudo caso-controle. Jornal de Pediatria 2001, 77: 294-98.

15. Bercini LO. Mortalidade neonatal em residentes em localidade urbana da região Sul do Brasil. Rev Saúde Pública 1994; 28: 38-45.

16. Guashino S. Assessement of perineonatal mortality and morbidity risk in twin pregnancy. Clin Exp Obstet Gynecol 1986; 1 (Supl1/2): 18-25.
17. Sociedade Brasileira de Pediatria. Educação médica continuada. Programa de Reanimação Neonatal. A importância do atendimento adequado ao recém-nascido na sala de parto. Disponível em <url: http://www.sbp.com.br> [2004 set 23].

18. Coutinho SB. Mortalidade neonatal em cinco maternidades da cidade do Recife em 1994: relatório final de pesquisa apresentado ao UNICEF. Recife: Editora: 1996.

19. Hübner ME, Ramirez R. Sobrevida, viabilidade y pronóstico del prematuro. Rev Med Chile 2002; 130: 931-38.

20. Castro ECM. Morbimotalidade hospitalar de recémnascidos de muito baixo peso no município de Fortaleza [dissertação mestrado]\}. Fortaleza: Departamento de Saúde Coletiva, Centro de Ciências da Saúde da Universidade Federal do Ceará; 2004.

21. Ferreira SMG. Sistema de Informação em Saúde. Belo Horizonte: Coopsmed; 2001. (Cadernos de Saúde, 4).

22. Ministério da Saúde. Fontes de informação. Indicadores básicos para a saúde no Brasil. Disponibilizado em <url:http://www.tabnet.datasus.gov.br>[2004 set 23].

23. National Committee on Vital and Health Statistics. Shaping a vision for 21 st century health statistics. Interim report, 2000. Disponível em <url:http://www.ncvhs.hns.gov> [2004 set 21].

24. Ministério da Saúde: Fundação Nacional de Saúde. Manual de procedimento do sistema de informação sobre mortalidade. Brasília (DF); 2001.

25. Ministério da Saúde. Fundação Nacional de Saúde. Manual de instruções para o preenchimento da declaração de óbito. 3. ed. Brasília (DF); 2001.

26. Aerts DRGC. Investigação dos óbitos perinatais e infantis: seu uso no planejamento de políticas públicas de saúde. J Pediatr (Rio J) 1997; 73: 29-36.

27. Fonseca SC, Coutinho ESF. Pesquisa sobre mortalidade perinatal no Brasil: revisão da metodologia e dos resultados. Cad Saúde Pública 2004; 20 (Supl 1): 7-19.

28. Vanderlei LC, Arruda BKG, Frias PG, Arruda S. Avaliação da qualidade de preenchimento das declarações de óbito em unidade terciária de atenção à saúde materno-infantil. Inf Epidemiol SUS 2002; 11: 7-14.

29. Novaes HMD, Almeida MF, Ortiz LP. Projeto Informação para Tomadores de Decisão em Saúde Pública - Tema: Gestão para redução da mortalidade infantil. 2. ed. São Paulo: BIREME, OPAS, Ministério da Saúde; 2004.

30. Sarinho SW, Coutinho SB, Acioli TML. Mortalidade neonatal em Recife, Pernambuco, 1998: causas básicas e grau de conhecimento dos neonatologistas acerca do preenchimento das Declarações de Óbito. Pediatria 2001; 23: 279-84.

31. Almeida MF, Mello Jorge MHP. O uso da técnica de linkage de sistemas de informação em estudos de coorte sobre mortalidade neonatal. Rev Saúde Pública 1996; 30: 141-7.

32. Morais Neto OLM, Barros MBA. Fatores de risco para mortalidade neonatal e pós-neonatal na Região CentroOeste do Brasil: linkage entre bancos de dados de nascidos vivos e óbitos infantis. Cad Saúde Pública 2000; 16: 47785 . 
33. Gould JB. Vital records for quality improvement. Pediatrics 1999; 103: 278-90.

34. Lansky S, França E, Leal MC. Mortes perinatais evitáveis em Belo Horizonte, Minas Gerais, Brasil, 1999. Cad Saúde Publica 2002; 18: 389-400.

35. Rosa MLG, Hortale VD. Óbitos perinatais evitáveis e estrutura de atendimento obstétrico na rede pública; estudo do caso de um Município da Região Metropolitana do Rio de Janeiro. Cad Saúde Pública 2000; 16: 773-83.

36. Goldani MZ, Barbieri MA, Bettiol H, Barbieri MR, Tomkins A. Mortalidade infantil e nível socioeconômico em uma cidade brasileira. Rev Saúde Pública 2001; 35: 256-261.

37. Frias PG. Análise de implantação do Projeto de Redução da MI em dois municípios de Pernambuco com semelhantes condições de vida [dissertação mestrado]. Recife: Departamento Materno-Infantil, Centro de Ciências da Saúde da Universidade Federal de Pernambuco; 2001.

38. Conselho Federal de Medicina. Pareceres. Brasília (DF); 2004. Disponível em <url:http://www.cfm.com.br>[2004 set 24].

39. Hartz ZMA, Champagne F, Leal MC, Contandriopoulos AP. Mortalidade infantil "evitável" em duas cidades do Nordeste do Brasil: indicador de qualidade do sistema local de saúde. Rev Saúde Pública 1996; 30: 310-8.

40. Formigli VLA, Silva LMV, Cerdeira AJP. Avaliação da atenção à saúde através da investigação de óbitos infantis. Cad. Saúde Pública 1996; 12 (Supl. 2): 33-41.

41. Santa Helena ET, Rosa MB. Avaliação da qualidade das informações relativas aos óbitos em menores de um ano em Blumenau, 1998. Rev Bras Saude Matern Infant 2003; 3: 75-83.

Recebido em 10 de junho de 2005

Versão final apresentada em 14 de setembro de 2005

Aprovado em 4 de novembro de 2005
42. Barros FC, Victora CG, Vaughan JP. Causas de mortalidade perinatal em Pelotas, RS (Brasil): utilização de uma classificação simplificada. Rev Saúde Publica 1987; 21: 310-6.

43. Menezes AMB, Victora CG, Barros FB. Estudo populacional de óbitos perinatais e infantis: metodologia, validade do diagnóstico e sub-registro. J Pediatr (Rio J) 1997; 73: 383-7.

44. Domingues RMSM, Santos EM, Leal MC. Aspectos da satisfação das mulheres com a assistência ao parto: contribuição para o debate. Cad Saúde Pública 2004; 20 (Supl 1): 52-62.

45. Ribeiro ERO, Barbieri MA, Bettiol H, Silva AAM. Comparação entre duas coortes de mães adolescentes em município do Sudeste do Brasil. Rev Saúde Pública 2000; 34: $136-42$.

46. Schramm JMA, Szwarcwald CL. Sistemas hospitalares como fonte de informação para estimar a mortalidade neonatal e a natimortalidade. Rev Saúde Pública 2000; 34 : 272-9.

47. Mello-Jorge MHP, Gotlieb SLD. O sistema de informação de atenção básica como fonte de dados para os sistemas de informações sobre mortalidade e sobre nascidos vivos. IESUS 2001; 10: 7-18.

48. Victora CG, Tomasi E. Análise de tendências temporais na mortalidade infantil de Alagoas, 1999-2002: relatório final para UNICEF. Pelotas, RS; 2004.

49. Ministério da Saúde. Secretaria de Atenção à Saúde. Manual dos Comitês de Prevenção do Óbito Infantil e Fetal. Brasília (DF); 2004. 